Article

\title{
Data Analytic Approaches for Mining Process Improvement-Machinery Utilization Use Case
}

\author{
Edyta Brzychczy ${ }^{1, *}$, Paulina Gackowiec ${ }^{1}\left[\right.$ and Mirko Liebetrau ${ }^{2}$ \\ 1 Faculty of Mining and Geoengineering, AGH University of Science and Technology, 30-059 Cracow, Poland; \\ gackowiec@agh.edu.pl \\ 2 Talpasolutions GmbH, 45327 Essen, Germany; mirko@talpasolutions.com \\ * Correspondence: brzych3@agh.edu.pl
}

Received: 5 December 2019; Accepted: 31 January 2020; Published: 7 February 2020

\begin{abstract}
This paper investigates the application of process mining methodology on the processes of a mobile asset in mining operations as a means of identifying opportunities to improve the operational efficiency of such. Industry 4.0 concepts with related extensive digitalization of industrial processes enable the acquisition of a huge amount of data that can and should be used for improving processes and decision-making. Utilizing this data requires appropriate data processing and data analysis schemes. In the processing and analysis stage, most often, a broad spectrum of data mining algorithms is applied. These are data-oriented methods and they are incapable of mapping the cause-effect relationships between process activities. However, in this scope, the importance of process-oriented analytical methods is increasingly emphasized, namely process mining (PM). PM techniques are a relatively new approach, which enable the construction of process models and their analytics based on data from enterprise IT systems (data are provided in the form of so-called event logs). The specific working environment and a multitude of sensors relevant for the working process causes the complexity of mining processes, especially in underground operations. Hence, an individual approach for event log preparation and gathering contextual information to be utilized in process analysis and improvement is mandatory. This paper describes the first application of the concept of $\mathrm{PM}$ to investigate the normal working process of a roof bolter, operating in an underground mine. By applying PM, the irregularities of the operational scheme of this mobile asset have been identified. Some irregularities were categorized as inefficiencies that are caused by either failure of machinery or suboptimal utilization of the same. In both cases, the results achieved by applying PM to the activity $\log$ of the mobile asset are relevant for identifying the potential for improving the efficiency of the overall working process.
\end{abstract}

Keywords: mining process; data mining; process mining; process improvement; BPM

\section{Introduction}

Introducing digitalization to the mining industry provides opportunities to improve productivity [1]. The acquisition of large amounts of machine data allows obtaining a more complete picture and in-depth knowledge of the efficiency in which processes are carried out. Hence, decision-making can be supported, and indications for processes efficiency improvement identified [2].

Mining companies aspire to continuously improve processes to increase the operational efficiency and safety of their personnel. Mining processes are characterized by very demanding and complex activities due to the challenging physical aspects (heat, cold, vibrations, noise) and the unpredictable conditions of work. In such environments, human errors, and defective equipment, as well as natural hazards, are serious risk factors. Risks in mining operations also arise from the use of heavy equipment and the occurrence of different types of energy (electrical, mechanical, other), which holistically 
contribute to a volatile environment [3]. Therefore, it is essential to accurately monitor the operational processes and mine-site equipment, comprehensively, and continuously, as well as to leverage insights that are generated by the monitoring solutions to support the decision-making process. The essence of environment and machinery monitoring in an underground mine is exemplified in [4,5].

Process data may derive from a variety of sources, for example, Enterprise Resource Planning (ERP) systems, machinery and device monitoring systems, the working environment, or employee location. The origins of different sources of data cause different degrees of granularity: from the most general (e.g., geometrical dimensions of the excavation), through more detailed operating states of machines (work, alarm, shutdown), for simple measurements (e.g., methane, currents in motors, switching on transformers). It is crucial that all such data are accounted to a specific process and they should be analyzed in this context, to provide for an in-depth efficiency and safety analysis.

Scientific publications suggest a variety of approaches for data analyses regarding various processes or working environment conditions. Most often, these approaches include a broad spectrum of data mining algorithms, used in the tasks of description [6-10], prediction [11-17], or classification of phenomena [18-22]. It is worth noting that these data-oriented methods are incapable of mapping the causal relationship between activities in the process to a degree where inefficiencies can be identified easily and on-point [23]. Therefore, in case process improvements are thought, it is mandatory to apply more process-oriented methods, e.g., Process Mining.

Process Mining (PM) originates in workflow analysis. Meanwhile, it is one of the most commonly used analytical approaches in Business Process Management (BPM). The tools that are applied in PM are various and they include techniques, like process model discovery, conformance checking, process model repair, role discovery, bottleneck analysis, or predicting the remaining flow time [24].

So far, individual cases of using this type of analysis in mining operations can be found. He et al. [25] reports on the application of PM to emergency rescue processes of fatal gas explosion accidents in China, while, in [26,27], the creation of event logs from a longwall monitoring system is presented.

Contemporary mining machinery is equipped with a wide variety of sensors. However, only a fraction of the data generated from these sensors is commonly utilized. The main reasons are the lack of a reliable and common system of data storage and analysis [28], as well as a lack of analytic expertise among process executors, and managers.

This paper presents the very first application of the PM approach for the analysis of mobile mining asset operation. The remainder of this paper is structured, as follows. In Section 2, to generate common basic understanding, introduction to selected concepts, such as the business process management, data mining, and process mining is presented. In this section, challenges related to PM implementation into the mining domain are also briefly described. The process mining analysis of roof bolter operation, based on the created event log, is presented in Section 3. The results of our analysis are discussed and the potentials for process improvement are identified. Finally, Section 4 concludes the paper and provides several directions for future work, pointing out the value of PM application in the effective management of mining machinery operations.

\section{Materials and Methods}

\subsection{Business Process Management}

This section will introduce the basics of Business Process Management (BPM) to achieve a general understanding of how process optimization is carried out. BPM has received considerable attention in recent years due to its potential for significantly increasing productivity and saving costs [29]. This discipline can be seen as an alignment of Information Technology (IT) infrastructure (e.g., data gathering systems, like an ERP tool) with current organizational demands of process improvement [30]. $\mathrm{BPM}$ is an iterative approach and it promotes the constant improvement of processes and procedures in organizations. 
The overall process architecture of the considered organization should be defined first in order to identify the critical processes. The evaluation of the processes can then be carried out with respect to its relevance to the whole process chain, as well as the identified degree of efficiency. This leads to a process hierarchy to most efficiently perform the process improvement.

BPM addresses different activities aiming for process improvement, generally presented as a cycle, referred to as the BPM cycle.

The BPM cycle consists of the following phases [31]:

- process model discovery,

- process analysis,

- process redesign,

- process implementation, and

- process monitoring and controlling.

Process model discovery helps to define a model that reflects how and in what sequence the processes are implemented in the organization ("as-is" model). At this stage, techniques for automatic model detection that are based on data can be used in addition to analyzing documentation, observations, and interviews. The current state of the process is documented in the form of one or several models.

Process analysis is carried out in order to identify errors that typically occur and lead to an extended duration or workload of the considered process. For this step, documentation and quantification concerning process effectiveness measures are crucial. In terms of the latter, various quantitative and qualitative analytic techniques are used (e.g., value-added analysis, why-why method, flow analysis, simulations [31]).

Process redesign suggests changes in the process that potentially reduce the identified errors (as investigated in the process analysis stage) and allow for the process sequence to be performed with improved efficiency. The effect of this stage is the "to-be" process model (redesigned, enhanced model).

Process implementation is the stage where the suggested changes or "to-be" process model is applied for the first time. Here, efficiency gains are actually generated for the first time.

The final stage is monitoring and controlling the process by analyzing data from the implemented process. It is investigated whether the changes have brought the assumed effects, and an overall higher process efficiency was achieved.

One can see that the process improvement requires process modeling and its analysis. PM can be used in these phases, as well as in the process monitoring and control phase. Our research focuses on the first two steps (process model discovery and process analysis) to point out inefficiencies and potential for optimization. Section 2.3 will present a characteristic of the PM approach.

\subsection{Data Mining-Data-Oriented Approach in Process Analytics}

Data mining is the exploration and analysis of large data sets to discover meaningful, previously unknown patterns and rules [32]. It is a component of a broader process, called knowledge discovery from databases (KDD), which also contains a selection of data, data pre-processing and cleaning, data transformation, as well as interpretation and evaluation phases [33].

Based on the kinds of investigated patterns, tasks in data mining can be classified into [34]: description, estimation, prediction, classification, clustering, and association.

Nowadays, the usefulness of various types of data mining algorithms for discovering new, potentially useful patterns and dependencies in data that can be used in the decision-making process are commonly known. However, their application is rather poor when it comes to process analysis (understood as a series of interrelated activities).

Among decision-makers and practitioners, managerial dashboards that descriptively present the "state" of the process are very popular [35]. On dashboards, most frequently, descriptive statistics, visualizations, and less frequently correlations are presented for information and decision-making 
purposes. Figure 1 presents an example dashboard, including process performance statistics for a roof bolter machine.

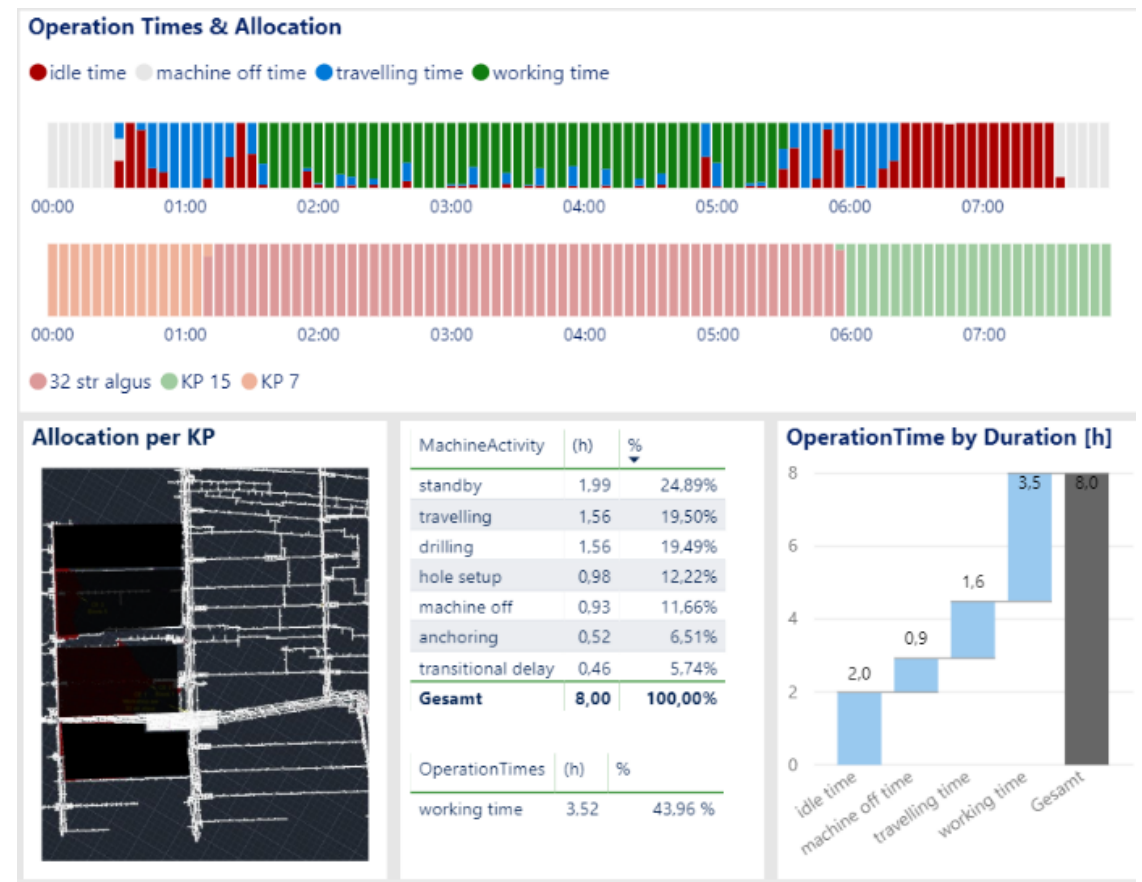

Figure 1. An example of dashboard showing machine performance and working process definition during an average working shift of a roof bolter in an underground mining operation [36].

The primary issue for creating such a report is to have sufficient data available to identify the activities occurring in the process, as well as defining their start and end time. Obtaining suitable data that are related to service processes or manufacturing processes from ERP systems is usually not a problem. However, the identification of activities must be carried out separately and usually requires expert domain knowledge in the case of processes carried out by mobile equipment in the mining industry. The identification of activities in the process most often involves algorithms that are formulated based on rules formulated by experts for selected variables. These variables or signals include e.g., rotational speed, current in engines, hydraulic pressures of different components, boom position and movement, and location in the excavation.

As can be seen in Figure 1, the general machine statuses have been defined as idle, machine off, traveling, or working (Figure 1 upper part). This classification gives a brief overview of the utilization and performance of the machine. Such classifications can usually be applied with basic data, as available from the engine control system [37].

The presented summary analytics, although they have undoubted practical advantages (simplicity, generalization of information), are static and they do not reflect the dynamic nature of the process as opposed to selected PM techniques. However, primary classification and generation of event logs are crucial for creating labeled data to apply PM techniques, as briefly explained in Section 2.4 and presented in Section 3.1.

\subsection{Process Mining_Process-Oriented Approach and Software in Process Analytics}

In recent years PM has become a very fast-growing discipline that focuses on the analysis of processes while using event data.

The underlying data structure used in PM is an event log, including defined data about process implementation. The three essential elements of an event log are case id, referring to a particular case, event/activity name, and timestamp of the event [38]. 
Table 1 presents an example of a simple event log.

Table 1. An example schematic event log.

\begin{tabular}{ccc}
\hline Case Id & Activity & Timestamp \\
\hline 1 & A & $01.04 .201700: 56: 15$ \\
1 & B & $01.04 .201701: 24: 25$ \\
1 & C & $01.04 .201701: 52: 14$ \\
1 & D & $01.04 .201701: 52: 35$ \\
1 & E & $01.04 .201701: 59: 21$ \\
\hline 2 & A & $01.04 .201702: 13: 35$ \\
2 & C & $01.04 .201702: 15: 17$ \\
2 & B & $01.04 .201702: 22: 06$ \\
2 & D & $01.04 .201702: 23: 08$ \\
2 & F & $01.04 .201702: 24: 31$ \\
\hline 3 & A & $01.04 .201702: 35: 17$ \\
3 & B & $01.04 .201702: 41: 44$ \\
3 & C & $01.04 .201702: 46: 09$ \\
3 & D & $01.04 .201702: 49: 09$ \\
3 & E & $01.04 .201702: 55: 37$ \\
\hline
\end{tabular}

Event logs can also store additional information, e.g., the name of the resource (person or device) executing the activity or other contextual data (e.g., size of an order, value of an invoice) [24].

One of the main tasks of PM is process model discovery, which involves transforming input data from IT systems supporting process into a model without using a priori information about the process. In addition to discovering process models from event logs, PM includes [39]:

- Conformance checking-based on comparing the existing model with actual event log records. This task allows for checking whether the process steps performed in the event log are consistent with the model and vice versa, while taking various types of models into account, including, for example, procedural, organizational, declarative, or business rules.

- Enhancement-based on an in-depth performance analysis of the implemented process by using contextual information recorded in the event log. This task is used to expand and improve the existing process model (e.g., by indicating process bottlenecks, capacity of individual resources, frequency of activities, loops analysis).

The PM tasks are presented in Figure 2.

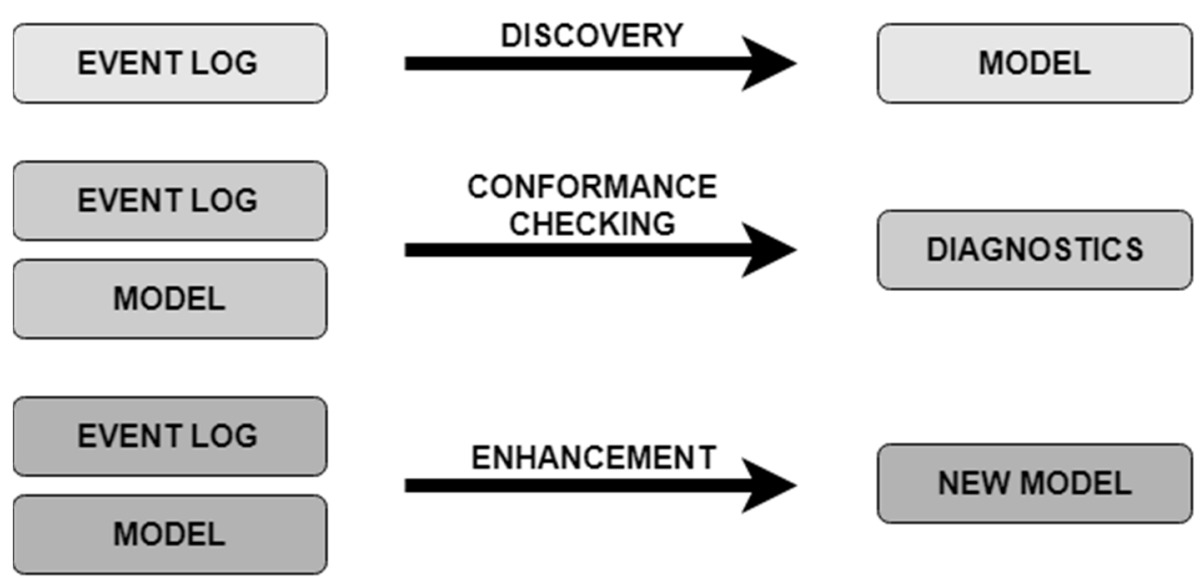

Figure 2. Process Mining (PM) tasks and corresponding outputs—based on [39].

In general, process models can be classified according to the degree of formality. The first group is informal models (e.g., Data Flow Diagram, Gantt chart). Their main purpose is to provide insights 
or support discussion, but cannot be used for enactment and rigorous analysis. The second group is formal models (e.g., Petri nets), allowing for deeper analysis and enactment. However, these models may be more difficult to construct than informal models. In practice, semi-formal models also exist (e.g., Business Process Model and Notation (BPMN), Unified Modelling Language (UML) activity diagrams, Event-driven Process Chain (EPCs)) [40].

The most basic process modeling notation is a transition system that consists of states and transitions that correspond to activities being executed [41]. The oldest and best investigated formal process models are Petri nets [42]. In the process modeling, a particular class of Petri nets is taken into consideration, namely, workflow net (WF-net) [43], which is the most popular construct in the process of model discovery. However, in practice, very often directly follows models are used.

Directly-follows models are variants of transitions systems and lie between transition systems and higher-level languages (e.g., Petri net). Their popularity stems from simplicity, the ability to express relationships that cannot be interpreted in a precise manner, and scalability [41]. Directly-follows models can be defined as a directed graph $G=(V, E)$. Vertices $\mathrm{V}$ denote the activities of a process. Directed edges $E \subseteq V \times V$ express that the target activity can be immediately executed after the source activity in a process instance [44]. An open graph might be extended by marking some vertices as start and completion vertices (Figure 3). However, unlike Petri nets, it is not able to express concurrency (see activity B and activity C relation in Figure 3).

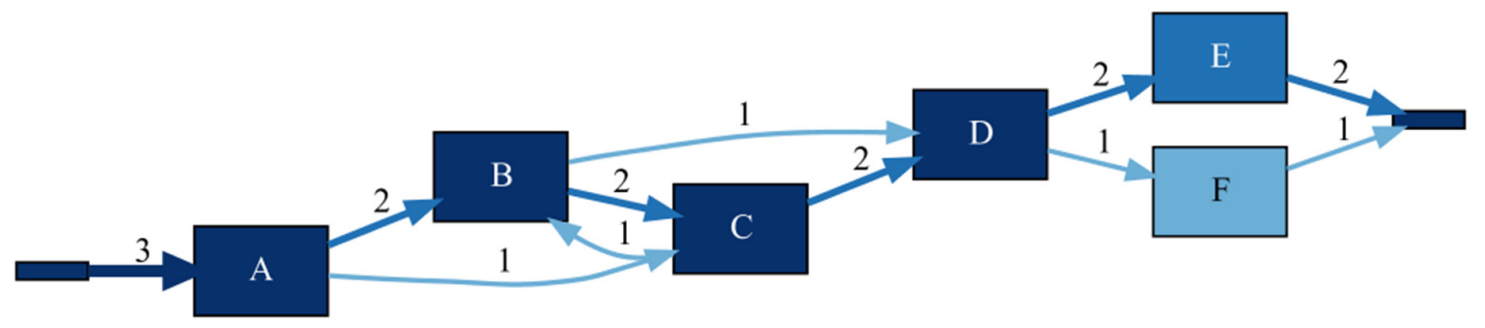

Figure 3. A directly-follows model for the event log presented in Table 1.

Discovering directly-follows models very often produces large process models that can be reduced with filtering of edges. Domain experts can easily interpret the simplistic models [41]. Directly-follows models are widely used in commercial software for PM, while Petri net and other modeling formalisms (e.g., BPMN, process trees) are mostly used in academic tools.

In 2018, Gartner released Market Guide for PM [45] with a description of 15 PM tools and their vendors. The majority of PM tools are of a commercial type. The most recognized tools include ProM (academic), Disco (Fluxicon), Celonis PM (Celonis), Minit (Minit), and ARIS PM (Software AG) [46].

All PM tools support process model discovery as well as performance analysis. Nevertheless, mainly academic tools (ProM [47], APROMORE [48]) support conformance checking as a result of formal models' usage in a process model discovery.

Performance analysis of the process includes, among others, time (how fast a process is executed), cost (how much a process execution costs), and quality (how well the process meets customer requirements and expectations). Existing PM tools support the analysis of entire processes or activities therein concerning performance measures, such as cycle time, processing time, and waiting time. They can pinpoint bottlenecks, resource underutilization (which are particularly useful in mining operations), and other performance issues that are observed over time [46]. Analysis of other process perspectives (cost, quality) due to process model enhancement depends on contextual information recorded in an event log. Very often, in this task, additional data mining techniques are used, i.e., classifiers [49].

A comprehensive overview of PM applications has been concluded in [50]. According to the authors, the most significant field for PM implementation is the industrial domain, which stems from the Industry 4.0 objectives of turning traditional manufacturing systems into Cyber-Physical Systems (CPS). Some use cases of PM in the context of manufacturing can be found in [51-56]. 


\subsection{Implementation of Process Mining into Mining Domain-Approach and Challenges}

The main challenge of PM implementation into the mining domain is the preparation of a suitable event $\log$, which reflects the process stages (activities) properly and enables analysis for valuable insights regarding the process improvement.

Nowadays, mining companies are well equipped with various IT systems (from SCADA to ERP class of solutions), supplying data pipeline with a different kind of information and data abstraction level; however, the crucial processes in a mining operation (related to technological operations) are mainly supported by low-level monitoring systems. A monitoring system can record hundreds of sensors or PLC readings (variables of binary or real type) with a short time interval (seconds, milliseconds); hence, the direct analysis of raw data is pointless from process analytics point of view.

PM implementation in the mining domain requires preparation phases, which are presented in Figure 4.

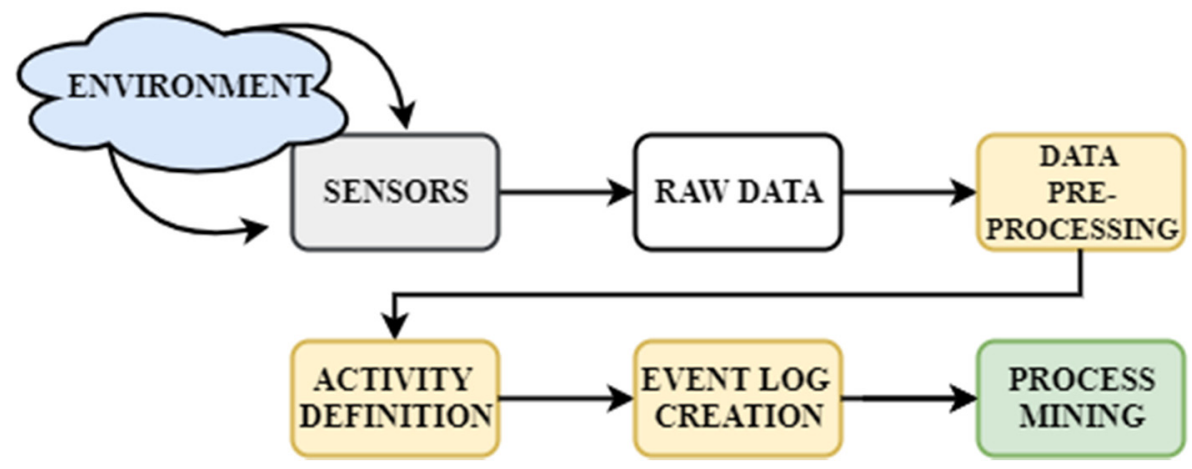

Figure 4. A general approach of PM implementation into mining domain.

The main preparation phases include data pre-processing, activity definition, and event log creation.

Mining environments, such as moisture, dust, temperature, vibrations, and others, very often influence sensor readings. Data pre-processing is needed to exclude outliers or abnormal behavior to utilize the data for identifying process steps or machine conditions (in addition to standard data quality checking). A commonly used quantitative approach for cleaning or smoothing data is the so-called robust regression [57]. The objective of this technique is to identify the impact of a data outlier on the whole data set. In case the relevance of such outliers is found to be insignificant, the course of the data set can be smoothened. The result of this technique is visualized on the basis of an exemplary data set displayed in Figure 5.

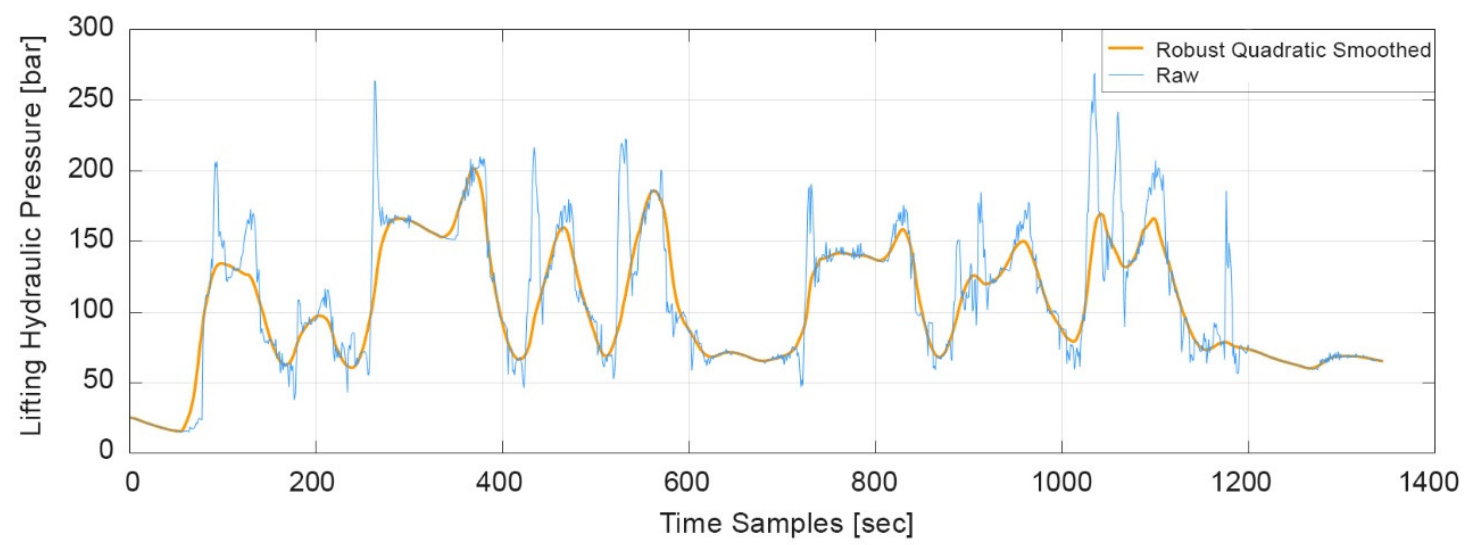

Figure 5. Result of Robust Quadratic Regression for data cleaning/smoothing of a hydraulics signal [36].

Robust Quadratic Regression was applied in the given case. Cleaned data are further used in the activity definition phase. 
The next step, activity definition phase can be seen as a transition from raw data to the events that represent the execution of activity in a process [58]. In the PM domain, such issue is known as event abstraction. In the same sense, human activity recognition (HAR) can be seen [59]. Various approaches for event abstraction can be used in this phase: unsupervised learning (e.g., clustering), supervised learning (e.g., classification with labeled data), behavioral patterns analysis, and others. A recent review on this subject is presented in [58].

In the case of mining processes, widely known approaches (e.g., clustering) are often failing because of the high variability of the process itself. There can be discovered states or activities (on various levels of abstraction), which cannot be easily interpreted, even by domain experts. On the other side, unsupervised techniques can bring interesting insights in the form of automatically discovered abnormal states in process realization, which can be helpful, e.g., for predictive maintenance purposes. For general process activity definition, supervised techniques seem to be more suitable due to clear rules for activity labeling provided by a domain expert.

A crucial issue that is related to the creation of an event log is its level of abstraction. A too general level (e.g., the definition of " working", " idle", " traveling", " not working") will not provide valuable findings for process improvement. On the other hand, a low level of event log abstraction can produce too complex and too detailed process models, thus making analysis difficult. The use of rule-based activity definition (labeling by experts) should guarantee the proper level of event log abstraction; however, it is strongly related to analytic needs and the involvement of domain experts. Dependency on the domain expert to provide the necessary information is one of the main limitations of the existing approaches in event log preparation. However, combining approaches from different areas (e.g., complex event processing) is seen as interesting future work of the PM community in the integration of separately developed ideas and techniques into more generic solutions that enable event abstraction [58]. Until then, the creation of a well-abstracted event log will rather be based on an adaptive approach in the mining domain, while taking process complexity and variability into consideration. The proposal of the adaptive approach for longwall mining, also dealing with other issues related to event log creation (e.g., lack of case id), was presented in [26].

In the next section, we present our results for PM application to the data of a roof bolter machine, starting with the description of our event log creation.

\section{Results}

\subsection{Process Description and Event Log Creation}

The roof bolter's primary objective is to secure the overlying strata of mining tunnels by installing rock bolts. The machine's status can always be defined as either "idle", " machine off", " traveling", or "working", as can be seen in Figure 6.

For a more detailed performance analysis, the process steps that are carried out in the machine status defined as "working" have been identified by utilizing corresponding characteristic machine data streams.

The machine status working is subdivided into the process steps: "anchoring", "transitional delay", "hole setup", and "drilling", all of which are only possible when the machine's drilling mode (opposite to driving mode) is activated, and the hydraulic support legs are extended.

The contained activities of working status are defined by the set of rules provided by a domain expert. Different relevant signals sourced from the machine are taken into account and pre-processed to achieve a suitable classification of activities. The activities are classified, as follows:

- Hole setup - before the hole can be drilled and directly after a bolt is installed, the boom needs to be positioned under the initial/next planned drill hole. This process is called the hole setup and it is defined by a number of hydraulic signals, which are indicating boom movement in different directions, as can be seen in Figure 7. 


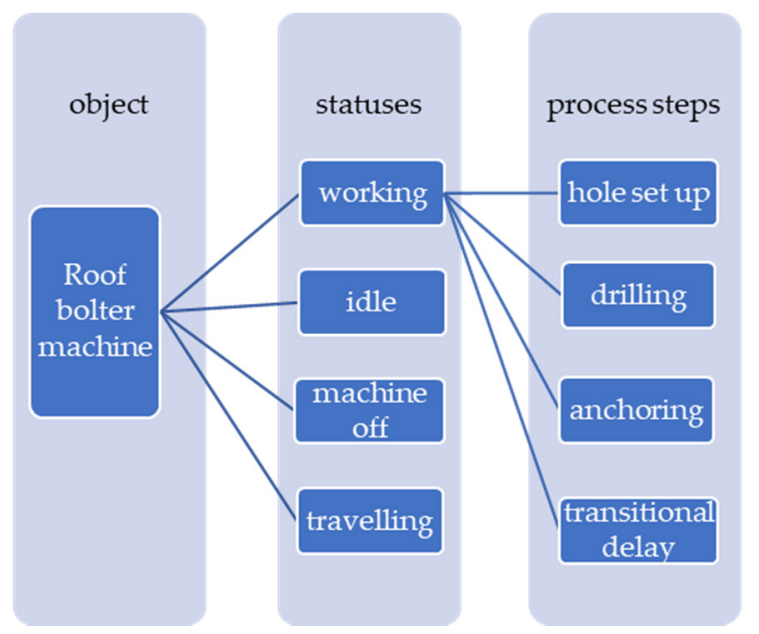

Figure 6. Statuses and process steps of the roof bolter operation.

- Drilling-once the boom is positioned correctly, the drilling process is the next production process. Thus, the rotating drill rod drills a hole into the roof and it is extracted by the machine after completion. Here, another set of hydraulic signals and mechanical sensors are taken into account, such as rpm of the drill itself and the position of the drill boom in respect to the anchor boom (Figure 8).

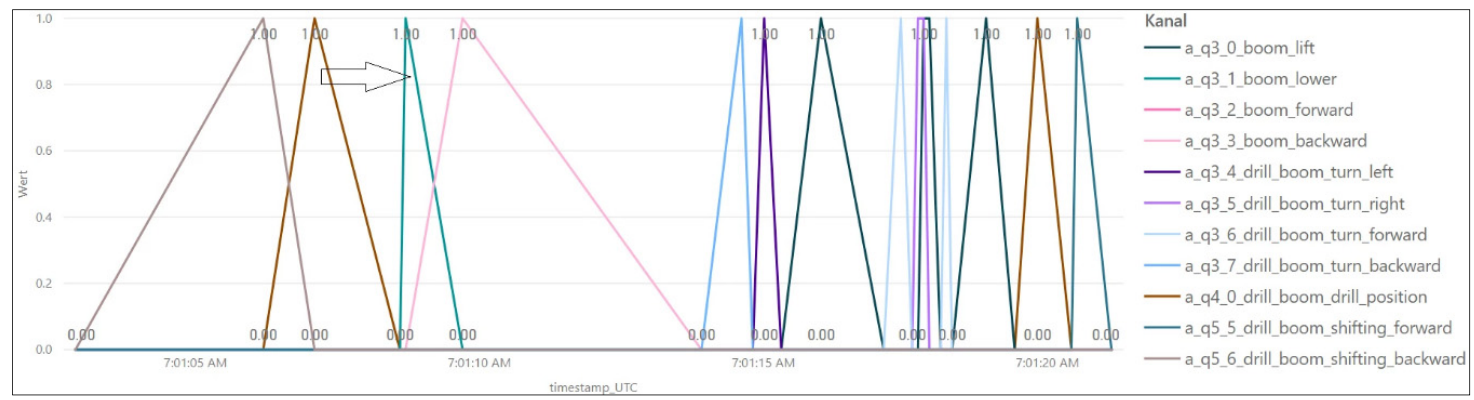

Figure 7. Explanatory signal sequence defined as "hole setup" activity [36].

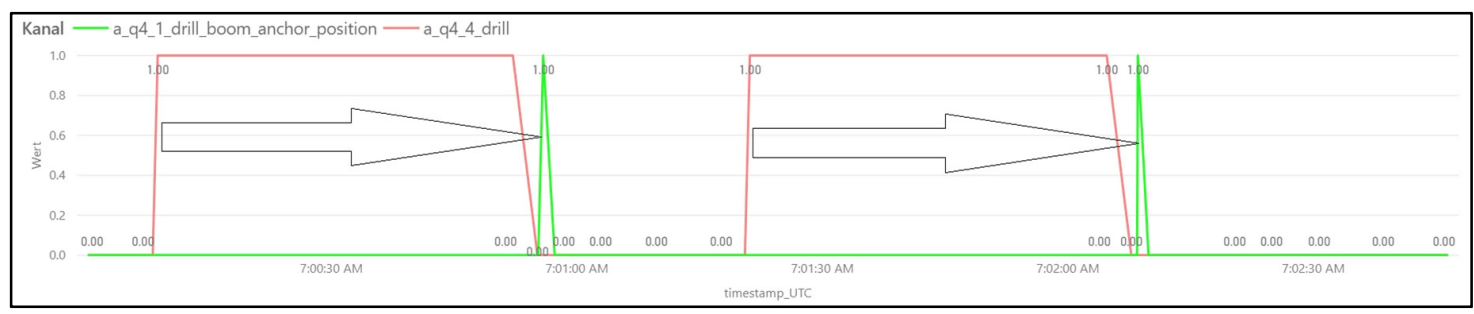

Figure 8. Specific signal sequence defined as "drilling" activity. Arrows indicate single drill events deriving from a combination of signals [36].

- Anchoring-after the drill hole is completed, an anchor is inserted automatically or at the operator's request into the hole, and torque is applied to secure the rock bolt. Again, different hydraulic sensors and the boom position are providing a signature like sequence to identify the activity (Figure 9).

- Transitional delay is the state when the machine's hydraulic system is switched on; however, no work is being performed (a.k.a. hydraulic standby). It can happen when manual work is required by the operator to continue production, e.g., changing the drilling rod/head or loading new bolts into the bolt magazine. Therefore, this process step is still considered to be a part of the machine status working. 


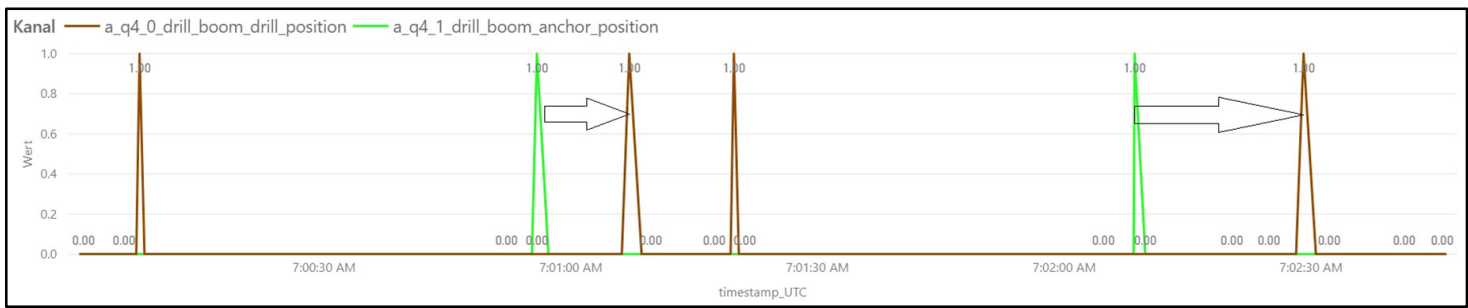

Figure 9. Specific signal sequence defined as "anchoring". Arrows indicate single anchoring events. In reference to Figure 7, two full drilling and bolting cycles can be derived [36].

Table 2 presents a fragment of the event log resulting from the classification described above. Case identification (id) is represented as shift id. Each shift starts/ends typically with "traveling", "idle", or "machine off" status.

Table 2. A fragment of roof bolter operation event log.

\begin{tabular}{cccc}
\hline Shift Id & Activity & $\begin{array}{c}\text { Timestamp } \\
\text { Activity Start }\end{array}$ & $\begin{array}{c}\text { Timestamp } \\
\text { Activity End }\end{array}$ \\
\hline$\ldots$ & $\ldots$ & $\ldots$ & $\ldots$ \\
1 & DRILLING & $2019-07-3122: 24: 15$ & $2019-07-31$ 22:24:55 \\
1 & TRANSITIONAL_DELAY & $2019-07-3122: 24: 56$ & $2019-07-3122: 24: 56$ \\
1 & ANCHORING & $2019-07-3122: 24: 57$ & $2019-07-3122: 25: 04$ \\
1 & TRANSITIONAL_DELAY & $2019-07-3122: 25: 05$ & $2019-07-3122: 25: 09$ \\
1 & HOLE_SETUP & $2019-07-3122: 25: 10$ & $2019-07-3122: 25: 10$ \\
1 & TRANSITIONAL_DELAY & $2019-07-3122: 25: 11$ & $2019-07-3122: 25: 11$ \\
1 & HOLE_SETUP & $2019-07-3122: 25: 12$ & $2019-07-3122: 25: 16$ \\
1 & TRANSITIONAL_DELAY & $2019-07-3122: 25: 17$ & $2019-07-3122: 25: 19$ \\
1 & DRILLING & $2019-07-3122: 25: 20$ & $2019-07-3122: 25: 58$ \\
1 & TRANSITIONAL_DELAY & $2019-07-3122: 25: 59$ & $2019-07-3122: 25: 59$ \\
1 & ANCHORING & $2019-07-3122: 26: 00$ & $2019-07-3122: 26: 08$ \\
1 & TRANSITIONAL_DELAY & $2019-07-3122: 26: 09$ & $2019-07-3122: 26: 11$ \\
1 & HOLE_SETUP & $2019-07-3122: 26: 12$ & $2019-07-3122: 26: 13$ \\
$\ldots$ & $\ldots$ & $\ldots$ & $\ldots$ \\
\hline
\end{tabular}

The analyzed event log contains a history of 34 shifts from a period of one month. The results of our PM analysis are presented in the next section.

\subsection{Process Modeling and Analysis with Process Mining}

In event log, 34 cases (each case is defined as different instance of the analyzed process) and 58021 events are recorded with the relative frequency, as follows: "transitional delay" $-44.4 \%$, "hole setup"-28.61\%, "drilling"- 9.3\%, "anchoring"-8.33\%, "idle"-4.06\%, "traveling"-3.17\%, and "machine off"-2.13\%.

Figure 10 shows the general view of the analyzed process (process map developed in Disco software $[60,61])$. 


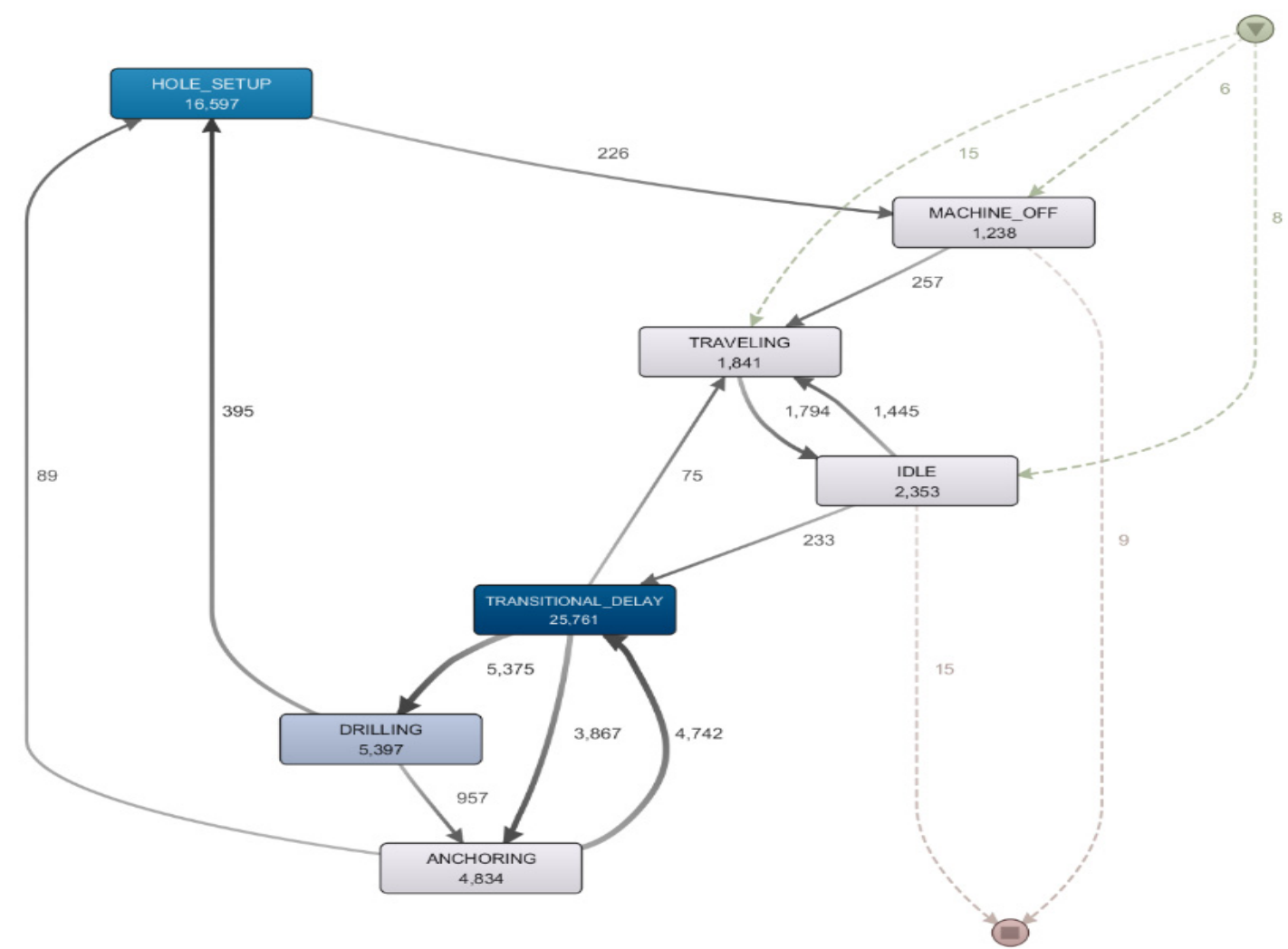

Figure 10. General process model of the roof bolter operation with absolute frequencies of activities.

This visualization identifies the most dominant paths in the process. The frequencies of the activities are displayed at the arcs as well as in the activities themselves. The process most frequently started with "traveling", so the machine has moved to the place where work is carried out. The start frequency for this activity equates to 15 cases. The most frequent end activity in the process is "idle" (15 cases).

By analyzing case duration in the process, the following findings can be confirmed: the longest-running case was performing for $7 \mathrm{~h} 29 \mathrm{~min}$. The shortest duration is $5 \mathrm{~h} 34 \mathrm{~min}$. The median execution time for cases in the data set is $6.8 \mathrm{~h}$. In the analyzed dataset within the existing variants, three specific times of day when cases started could be identified: 5:00 am, 1:00 pm, and 9:00 pm, which correspond to the times of the shifts beginning in the flexible shift system.

To make the analysis more accurate, all of the paths of the workflow have been revealed, and the result is presented in Figure 11.

In total, the most frequent activities in the process are "hole setup" and "transitional delay". Attention should be paid to the second of the mentioned activities, which is executed most often in the analyzed process, whereas, in terms of efficiency, it is unfavorable, because it does not provide any added value in the process. In the process map, the most frequent paths in the process between "transitional delay" and "hole setup", as well as the loop with these activities are to be pointed out.

Based on detailed process visualization, some exceptions to the standard/regular process workflow sequence could be identified. In Figure 11, three shifts in the dataset are shown that started with the "hole setup" activity. These examples may be considered as peculiar in the usual process.

Additionally, the analysis was carried out from a performance point of view to gain detailed knowledge regarding process execution. The performance metrics of the process consider cumulated duration for performing each activity as well as total delays on each path. Visualization of the process performance enables an analysis of parameters such as, e.g., minimum, maximum, or mean values of duration between activities, which are marked as red arrows. In the process map, the most intense coloring has highlighted the longest durations. As could be noticed based on the total duration of 
activities (Figure 12), the most time-consuming activities in the process are "drilling" and "traveling", for which the accumulated durations over all cases in the concerned period are about 54 and $46 \mathrm{~h}$, respectively. The shortest duration is determined for activity "anchoring", for which the aggregated duration equals $15.8 \mathrm{~h}$.

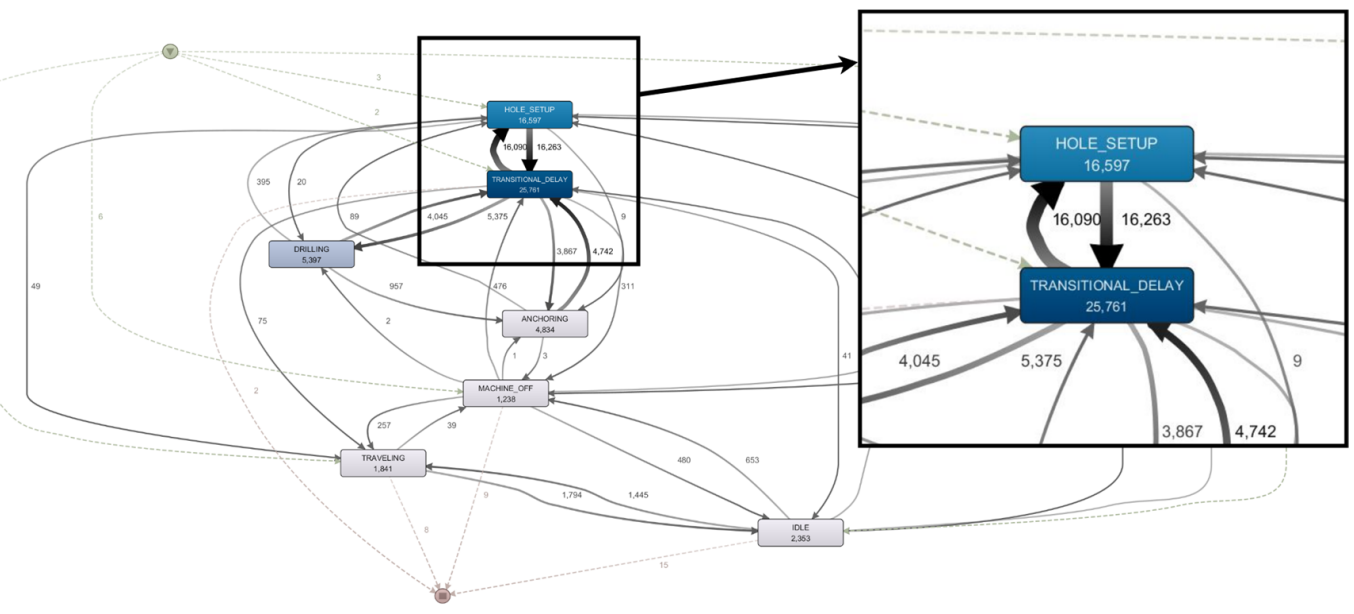

Figure 11. Detailed process map of the roof bolter operation with absolute frequencies of activities (in Disco software).

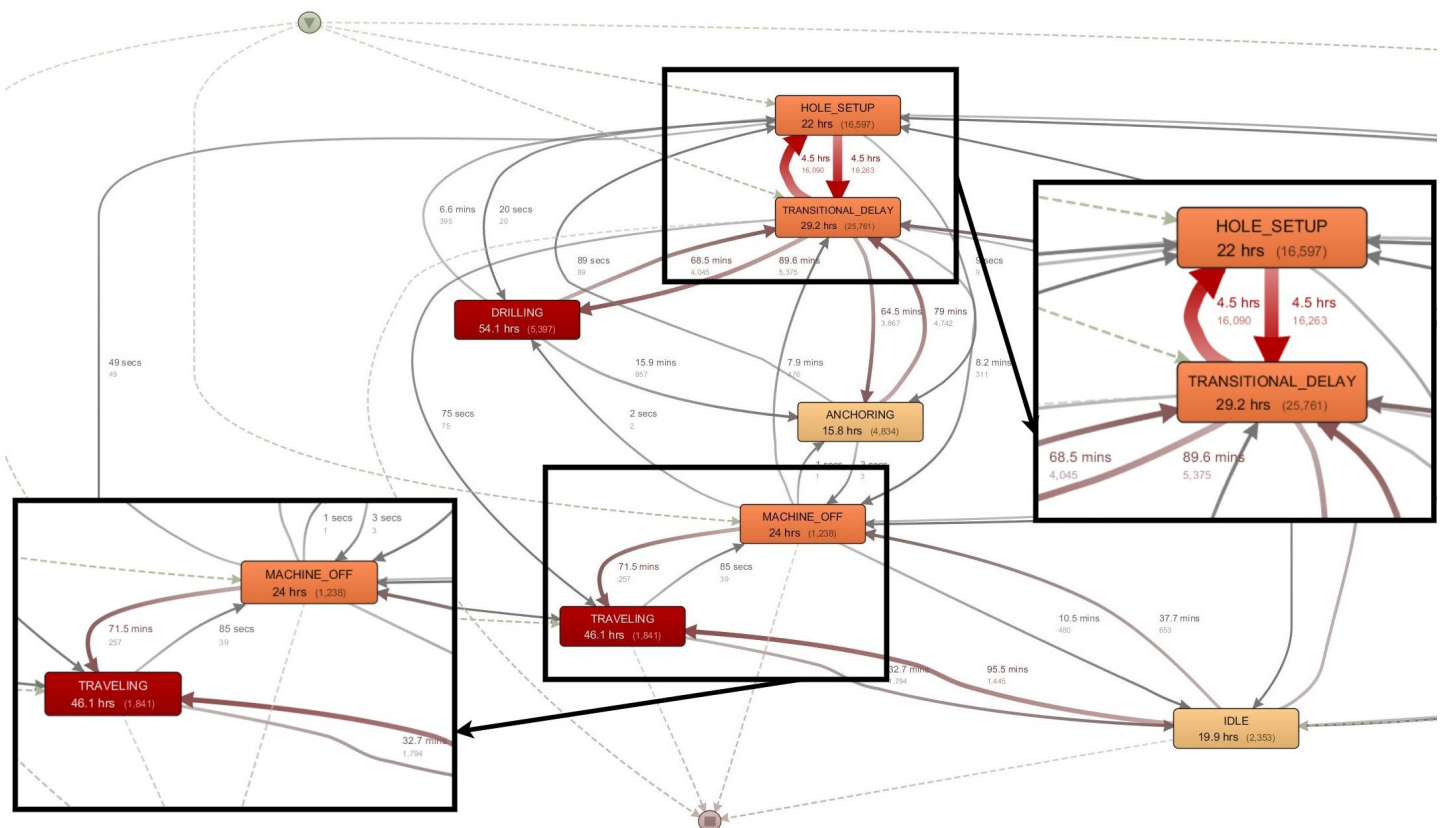

Figure 12. Process model of the roof bolter operation with total duration metric (in Disco software).

Activities "drilling" and "anchoring", as well as "hole setup", are the main activities in the roof bolter operation. Their existence, frequency, and duration are understandable. However, the analysis of the performance process map found that the high-impact area in a process are paths connected with step "transitional delay", which takes around $29 \mathrm{~h}$ in total. This delay is related to manual operations in the process, which cannot be classified by machine data. However, the necessity of changing drill bits and re-charging bolts for a steady process is evident. The time that is spent in "transitional delay" does not contain any type of idling during the working state of the machine. Regarding the efficiency increase, the reduced share of transitional delay with respect to total working time towards a potential minimum should be taken into consideration. 
Figure 13 presents process visualization with the most extended execution times. A secondary metric displayed in the map is absolute frequency. The most important areas of the process are highlighted in the process map.

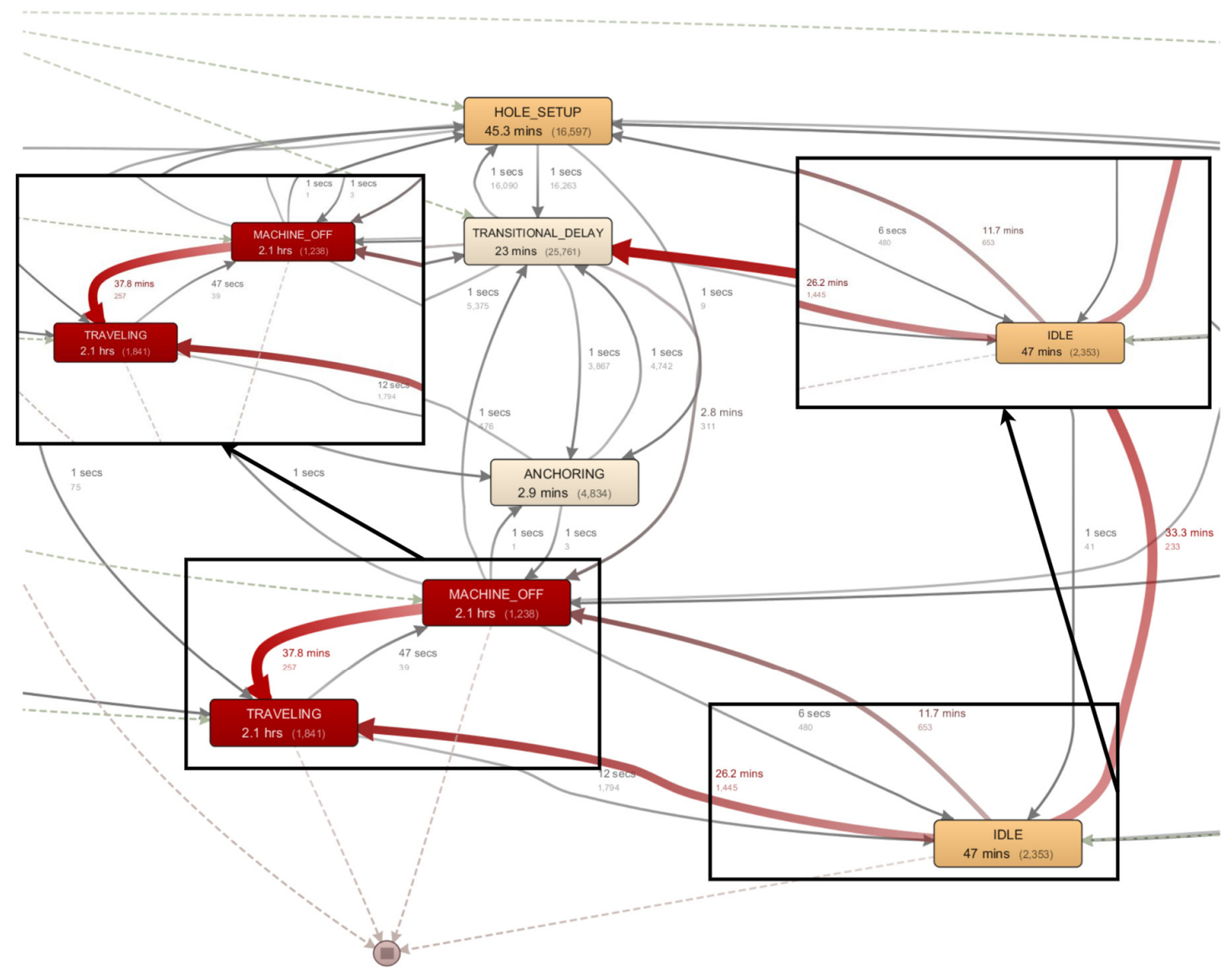

Figure 13. Detailed process map of the roof bolter operation with maximal duration metric (in Disco software).

The process map illustrates that the longest duration time could be identified not only for "traveling" but also for the "machine off" state. Regarding the most frequent activities, the maximum duration of "transitional delay" is $23 \mathrm{~min}$ and, for "hole setup", equals to $45.3 \mathrm{~min}$.

Events that can be easily visualized with the applied technique do point out inefficiencies or operational failures of the machine. Therefore, it can be used to investigate the occurrences of extended process step durations and reduce the risk for a frequent delay of the overall process.

Statistical analyses of case/activity distribution over time, variants of the process execution, as well as a handover of work (with social network analysis) or resource utilization over time, can provide additional information. However, further analysis requires contextual information regarding process resources (i.e., employees, IT systems).

\section{Discussion and Conclusions}

Being aware of the necessity of innovation and continuous improvement of processes, mining companies are moving towards modern solutions and automation of mining operations. Terms, such as intelligent mine, Industry 4.0, Industrial Internet of Things (IIoT), real-time monitoring, and data analysis, have been widely discussed and well-established among researchers, as well as mining practitioners [62]. Based on [63] for the paradigm of Industry 4.0, one of the key pillars is big data and analytics. Attention should be paid to analytical methods, which enable not only 
data-oriented analysis, but primarily the possibility to have insight into processes and turn gathered information into valuable knowledge by identifying process inefficiencies and deviations.

The necessary steps for identifying inefficiencies in processes, according to the BPM cycle, are process modeling and its analysis. In these stages, a wide range of supporting techniques can be applied. For these purposes, we presented PM as a new analytic approach in the mining domain. The application of PM in mining operations, especially in machine-driven processes, extends the possibility of sensor data usage, aiming for better process understanding and identification of process inefficiencies. Once inefficiencies are identified, they should be subject to further investigation, which results in operational changes, according to the BPM cycle (process redesign and implementation).

We presented an analysis of an example mining process as a case study with selected PM techniques. Analysis performed on roof bolter operation has enabled:

- identification of possible non-compliant behavior during process execution (i.e., shifts started with unusual activities);

- identification of high impacts area in process execution (repetitions and loops between activities-i.e., "transitional delay" - "hole setup"); and,

- identification of time lost in the process (based on activity duration statistics, duration of time between activities, especially in working operation time).

The presented results have initially shown the potential of process-oriented analytics in this area. The identified cases supported by contextual information provide decision-makers with opportunities to apply changes for improving efficiency (e.g., organizational changes, like work standardization; technological changes, like loop or repetition limitation).

The increase in efficiency and competitiveness of mining companies with PM analysis will probably be advantageous. With a process-centric approach, mining companies can identify process deviations, and bottlenecks, which are based on event logs from system databases. Using specialized, industrial knowledge, companies can interpret process models and maximize productivity and performance properly. Once sufficient activity log data has been evaluated and the PM technique is applied in an automated manner, notifications on inefficiencies or delays in process chains can be generated without manually setting normal ranges.

The power of PM techniques lays in the reflection of real process behavior recorded in IT systems. Thus, the obtained process models can be treated as more reliable than process observations and complementary to other manual process documentation sources.

The mining community has still not discovered PM. Despite raised challenges related to the creation of relevant event logs in the mining domain, PM might become a new useful analytic for accelerating process improvement and efficiency. Further, it could play an important role in automated notification schemes for process and performance monitoring without applying expert knowledge or manual input. Thus, informing relevant stakeholders on irregularities, failures, and delays, enabling them to react on identified problems, giving the inputs to increase efficiency of production processes.

As for our future research, the PM application to activity logs of less complex processes, such as loading-hauling-dumping of dump trucks or LHDs/wheel loaders, will most certainly result in an even more in-depth understanding and faster identification of inefficiencies.

Author Contributions: Conceptualization, E.B. and M.L.; methodology, E.B.; formal analysis, E.B. and P.G.; data curation, M.L.; writing-original draft preparation, E.B. and P.G.; writing-review and editing, E.B. and M.L.; visualization, P.G.; supervision, E.B.; project administration, M.L.; funding acquisition, E.B., M.L. All authors have read and agreed to the published version of the manuscript.

Funding: This research was funded by EIT Raw Materials, grant number 18327-project SmartHUB - smart courier field data IoT radio network \& big data analytics. (eit) RawMaterials

Acknowledgments: Authors would like to thank kindly Lee Bailey, Charlotte Schmitz, Ayoub Essourni, Jens Winkelman, and Timo Boße of talpasolutions $\mathrm{GmbH}$ for their work on event logs, data mining and data provision. 
Conflicts of Interest: The authors declare no conflict of interest.

\section{References}

1. Lööw, J.; Abrahamsson, L.; Johansson, J. Mining 4.0-The Impact of New Technology from a Work Place Perspective. Mining Metall. Explor. 2019, 36, 701-707. [CrossRef]

2. Ghattas, J.; Soffer, P.; Peleg, M. Improving business process decision making based on past experience. Decision Support Syst. 2014, 59, 93-107. [CrossRef]

3. Lööw, J.; Nygren, M. Initiatives for increased safety in the Swedish mining industry: Studying 30 years of improved accident rates. Saf. Sci. 2019, 117, 437-446. [CrossRef]

4. Wojaczek, A.; Wojaczek, A.K. Monitoring the environment and machines in underground mine. Zesz. Nauk. Inst. Gosp. Sur. Miner. Energ. PAN. 2017, 99, 57-70.

5. Wojaczek, A.; Wojaczek, A.K. Systemy monitoringu w kopalni podziemnej. Syst. Support. Prod. Eng. 2016, 1, 526-536.

6. Jonek-Kowalska, I.; Turek, M. Dependence of total production costs on production and infrastructure parameters in the polish hard coal mining industry. Energies 2017, 10, 1480. [CrossRef]

7. Qiao, W.; Liu, Q.; Li, X.; Luo, X.; Wan, Y.L. Using data mining techniques to analyze the influencing factor of unsafe behaviors in Chinese underground coal mines. Resour. Policy 2018, 59, 210-216. [CrossRef]

8. Kopacz, M. The impact assessment of quality parameters of coal and waste rock on the value of mining investment projects - hard coal deposits. Miner. Resour. Manag. 2015, 31, 161-188. [CrossRef]

9. Bongers, D.R.; Gurgenci, H. Fault Detection and Identification for Longwall Machinery Using SCADA Data. In Complex System Maintenance Handbook. Springer Series in Reliability Engineering; Springer: London, UK, 2008; Volume 8, pp. 611-641.

10. Elsayed, E.A. Reliability Prediction and Accelerated Testing; In Complex System Maintenance Handbook. Springer Series in Reliability Engineering; Springer: London, UK, 2008; Volume 8.

11. Al-Chalabi, H.; Ahmadzadeh, F.; Lundberg, J.; Ghodrati, B. Economic lifetime prediction of a mining drilling machine using an artificial neural network. Int. J. Mining Reclam. Environ. 2014, 28, 311-322. [CrossRef]

12. Bodlak, M.; Kudełko, J.; Zibrow, A. Machine Learning in predicting the extent of gas and rock outburst. E3S Web Conf. 2018, 71. [CrossRef]

13. Moczulski, W. A new decision support tool for fault diagnosis, hazard prediction and analysis in mining industry-a case study. In Proceedings of the 24th World Mining Congress, Rio de Janeiro, Brazil, 18-21 October 2016; pp. 38-48.

14. Mahdevari, S.; Shahriar, K.; Sharifzadeh, M.; Tannant, D.D. Stability prediction of gate roadways in longwall mining using artificial neural networks. Neural Comput. Appl. 2017, 28, 3537-3555. [CrossRef]

15. Boullé, M. Predicting dangerous seismic events in coal mines under distribution drift. In Proceedings of the 2016 Federated Conference on Computer Science and Information Systems (FedCSIS), Gdansk, Poland, 11-14 September 2016; pp. 221-224.

16. Deb, D.; Kumar, A.; Rosha, R.P.S. Forecasting shield pressures at a longwall face using artificial neural networks. Geotech. Geol. Eng. 2006, 24, 1021-1037. [CrossRef]

17. Verma, A.K.; Kishore, K.; Chatterjee, S. Prediction Model of Longwall Powered Support Capacity Using Field Monitored Data of a Longwall Panel and Uncertainty-Based Neural Network. Geotech. Geol. Eng. 2016, 34, 2033-2052. [CrossRef]

18. Gernand, J. Machine Learning Classification Models for More Effective Mine Safety Inspections. In Proceedings of the ASME 2014 International Mechanical Engineering Congress and Exposition, Montreal, QC, Canada, 14-20 November 2014; Volume 14.

19. Hargrave, C.O.; James, C.A.; Ralston, J.C. Infrastructure-based localisation of automated coal mining equipment. Int. J. Coal Sci. Technol. 2017, 4, 252-261. [CrossRef]

20. Jamróz, D.; Niedoba, T. Application of multidimensional data visualization by means of self-organizing Kohonen maps to evaluate classification possibilities of various coal types. Arch. Min. Sci. 2015, 60, 39-50.

21. Jedliński, Ł.; Gajewski, J. Optimal selection of signal features in the diagnostics of mining head tools condition. Tunn. Undergr. Sp. Technol. 2019, 84, 451-460. [CrossRef] 
22. Zhou, J.; Li, X.; Mitri, H.S.; Wang, S.; Wei, W. Identification of large-scale goaf instability in underground mine using particle swarm optimization and support vector machine. Int. J. Min. Sci. Technol. 2013, 23, 701-707. [CrossRef]

23. van der Aalst, W. Process Mining: Overview and Opportunities. ACM Trans. Manag. Inf. Syst. 2012, 3, 7:1-7:17. [CrossRef]

24. van der Aalst, W.M.P. Process mining in the large: A tutorial. Lect. Notes Bus. Inf. Process. 2014, 172, 33-76.

25. He, Z.; Wu, Q.; Wen, L.; Fu, G. A process mining approach to improve emergency rescue processes of fatal gas explosion accidents in Chinese coal mines. Saf. Sci. 2019, 111, 154-166. [CrossRef]

26. Brzychczy, E.; Trzcionkowska, A. Creation of an event log from a low-level machinery monitoring system for process mining purposes. In Intelligent Data Engineering and Automated Learning-IDEAL 2018; Yin, $\mathrm{H}$., Camacho, D., Novais, P., Tallón-Ballesteros, A.J., Eds.; Springer Nature: Cham, Switzerland, 2018; pp. 54-63.

27. Brzychczy, E.; Trzcionkowska, A. Process-Oriented Approach for Analysis of Sensor Data from Longwall Monitoring System. In Intelligent Systems in Production Engineering and Maintenance; Burduk, A., Chlebus, E., Nowakowski, T., Tubis, A., Eds.; Springer International Publishing: Cham, Switzerland, 2019; pp. 611-621.

28. David McHutchon, Industry 4.0 in Mining —Challenges and Opportunities. Available online: https://www. minexforum.com/en/industry-4-0-in-mining-challenges-and-opportunities/ (accessed on 25 September 2019).

29. van der Aalst, W.; Zhao, J.L.; Wang, H. Editorial: Business Process Intelligence: Connecting Data and Processes. ACM Trans. Manag. Inf. Syst. 2015, 5. [CrossRef]

30. Maita, A.R.C.; Martins, L.C.; López Paz, C.R.; Rafferty, L.; Hung, P.C.K.; Peres, S.M.; Fantinato, M. A systematic mapping study of process mining. Enterp. Inf. Syst. 2018, 12, 505-549. [CrossRef]

31. Dumas, M.; La Rosa, M.; Mendling, J.; Reijers, H.A. Fundamentals of Business Process Management; Springer: Berlin/Heidelberg, Germany, 2013; ISBN 9783642331428.

32. Pujari, A.K. Data Mining Techniques; Universities Press: Hyderguda, India, 2001; ISBN 8173713804.

33. Azevedo, A. Data Mining and Knowledge Discovery in Databases. In Advanced Methodologies and Technologies in Network Architecture, Mobile Computing, and Data Analytics; M. Khosrow-Pour, D.B.A., Ed.; IGI Global: Hershey, PA, USA, 2019; pp. 502-514.

34. Larose, D.T.; Larose, C.D. Discovering Knowledge In Data: An Introduction to Data Mining, 2nd ed.; Wiley: Hoboken, NJ, USA, 2014; ISBN 9780470908747.

35. zur Muehlen, M.; Shapiro, R. Business Process Analytics. In Handbook on Business Process Management 2: Strategic Alignment, Governance, People and Culture; vom Brocke, J., Rosemann, M., Eds.; Springer: Berlin/Heidelberg, Germany, 2015; pp. 243-263.

36. Internal Documentation of Talpasolutions GmbH. Talpasolutions GmbH; Essen, Germany, Unpublished work; 2018.

37. Krot, P.; Sliwinski, P.; Zimroz, R.; Gomolla, N. The identification of operational cycles in the monitoring systems of underground vehicles. Measurement 2020, 151, 107-111. [CrossRef]

38. van der Aalst, W. Process Mining: Data Science in Action; Springer-Verlag: Berlin/Heidelberg, Germany, 2016; ISBN 9783662498514.

39. van der Aalst, W.; Adriansyah, A.; de Medeiros, A.K.A.; Arcieri, F.; Baier, T.; Blickle, T.; Bose, J.C.; van den Brand, P.; Brandtjen, R.; Buijs, J.; et al. Process Mining Manifesto. Business Process Management Workshops; Daniel, F., Barkaoui, K., Dustdar, S., Eds.; Springer: Berlin/Heidelberg, Germany, 2012; pp. 169-194.

40. van der Aalst, W.M.P. Process-Aware Information Systems: Lessons to Be Learned from Process Mining. In Transactions on Petri Nets and Other Models of Concurrency II: Special Issue on Concurrency in Process-Aware Information Systems; Jensen, K., van der Aalst, W.M.P., Eds.; Springer: Berlin/Heidelberg, Germany, 2009; pp. 1-26.

41. Leemans, S.J.J.; Poppe, E.; Wynn, M.T. Directly Follows-Based Process Mining: Exploration \& a Case Study. In Proceedings of the 2019 International Conference on Process Mining (ICPM), Aachen, Germany, 24-26 June 2019; pp. 25-32.

42. van der Aalst, W.M.P. Decomposing Petri Nets for Process Mining: A Generic Approach. Distrib. Parallel Databases 2013, 31, 471-507. [CrossRef]

43. van Der Aalst, W.M.P.; Van Hee, K.M.; Ter Hofstede, A.H.M.; Sidorova, N.; Verbeek, H.M.W.; Voorhoeve, M.; Wynn, M.T. Soundness of workflow nets: Classification, decidability, and analysis. Form. Asp. Comput. 2011, 23, 333-363. [CrossRef] 
44. Bauer, M.; Senderovich, A.; Gal, A.; Grunske, L.; Weidlich, M. How Much Event Data Is Enough? A Statistical Framework for Process Discovery. In Advanced Information Systems Engineering. CAiSE 2018; Krogstie, J., Reijers, H., Eds.; Springer: Cham, Switzerland, 2018; pp. 239-256.

45. Kerremans, M. Market Guide for Process Mining. Gartner 2019, 1-33.

46. Bru, F.; Claes, J. The perceived quality of process discovery tools. arXiv 2018, arXiv:1808.06475. Available online: https://arxiv.org/ftp/arxiv/papers/1808/1808.06475.pdf (accessed on 1 February 2020).

47. Claes, J.; Poels, G. Process Mining and the ProM Framework: An Exploratory Survey. In Proceedings of the Business Process Management Workshops; La Rosa, M., Soffer, P., Eds.; Springer: Berlin/Heidelberg, Germany, 2013; pp. 187-198.

48. La Rosa, M.; Reijers, H.A.; Van Der Aalst, W.M.P.; Dijkman, R.M.; Mendling, J.; Dumas, M.; García-Bañuelos, L. APROMORE: An advanced process model repository. Expert Syst. Appl. 2011, 38, 7029-7040. [CrossRef]

49. Nguyen, H.; Dumas, M.; ter Hofstede, A.H.M.; La Rosa, M.; Maggi, F.M. Business Process Performance Mining with Staged Process Flows. In Advanced Information Systems Engineering; Nurcan, S., Soffer, P., Bajec, M., Eder, J., Eds.; Springer International Publishing: Cham, Switzerland, 2016; pp. 167-185.

50. Dees, M.; de Leoni, M.; Mannhardt, F. Enhancing Process Models to Improve Business Performance: A Methodology and Case Studies. In On the Move to Meaningful Internet Systems. OTM 2017 Conferences; Panetto, H., Debruyne, C., Gaaloul, W., Papazoglou, M., Paschke, A., Ardagna, C.A., Meersman, R., Eds.; Springer International Publishing: Cham, Switzerland, 2017; pp. 232-251.

51. dos Santos Garcia, C.; Meincheim, A.; Faria Junior, E.R.; Dallagassa, M.R.; Sato, D.M.V.; Carvalho, D.R.; Santos, E.A.P.; Scalabrin, E.E. Process mining techniques and applications-A systematic mapping study. Expert Syst. Appl. 2019, 133, 260-295. [CrossRef]

52. Rozinat, A.; de Jong, I.S.M.; Günther, C.W.; van der Aalst, W.M.P. Process mining applied to the test process of wafer scanners in ASML. IEEE Trans. Syst. Man Cybern. Part C Appl. Rev. 2009, 39, 474-479. [CrossRef]

53. Liu, T.; Yi, H.; Ni, Z.; Liu, X. A closed-loop workflow management technique based on process mining. In Proceedings of the 15th International Conference on Mechatronics and Machine Vision in Practice, Auckland, New Zealand, 2-4 December 2008; pp. 435-440.

54. Ho, G.T.S.; Lau, H.C.W. Development of an olap-fuzzy based process mining system for quality improvement. IFIP Int. Fed. Inf. Process. 2006, 228, 243-258.

55. Lee, S.K.; Kim, B.; Huh, M.; Cho, S.; Park, S.; Lee, D. Mining transportation logs for understanding the after-assembly block manufacturing process in the shipbuilding industry. Expert Syst. Appl. 2013, 40, 83-95. [CrossRef]

56. Myers, D.; Suriadi, S.; Radke, K.; Foo, E. Anomaly detection for industrial control systems using process mining. Comput. Secur. 2018, 78, 103-125. [CrossRef]

57. Cleveland, W.S. Robust Locally Weighted Regression and Smoothing Scatterplots. J. Am. Stat. Assoc. 1979, 74, 829-836. [CrossRef]

58. Diba, K.; Batoulis, K.; Weidlich, M.; Weske, M. Extraction, correlation, and abstraction of event data for process mining. WIREs Data Mining Knowl Discov. 2019, e1346. [CrossRef]

59. Riboni, D.; Murtas, M. Sensor-based activity recognition: One picture is worth a thousand words. Future Gen. Computer Syst. 2019, 101, 709-722. [CrossRef]

60. Günther, C.W.; Rozinat, A. Disco: Discover your processes. In Proceedings of the Demonstration Track of the 10th International Conference on Business Process Management, BPM Demos 2012, Tallinn, Estonia, 4 September 2012; pp. 40-44.

61. Disco User Guide. Available online: https://fluxicon.com/book/read/reference/ (accessed on 15 October 2019).

62. Żyrek, L.; Zasadni, W.; Lubryka, J.; Szołtysik, R. ENDIS 4.0 as replay on requirements of Industry 4.0 in field of power supply and automation for mining. Min. - Informatics, Autom. Electr. Eng. 2018, 533, 95-100. [CrossRef]

63. Vaidya, S.; Ambad, P.; Bhosle, S. Industry 4.0-A Glimpse. Procedia Manuf. 2018, 20, 233-238. [CrossRef] 